\title{
Control y mejora de la coordinación entre asignaturas de una titulación universitaria
}

\author{
Eva M. Ortigosa ${ }^{a}$, Ester Martín Garzón ${ }^{b}$, Pilar M. Ortigosab, Joaquín Ortega \\ Casanova $^{c}$ y Luis F. Romeroc \\ a Universidad de Granada, ortigosa@ugr.es, b Universidad de Almería, gmartin@ual.es, \\ ortigosa@ual.es y c Universidad de Málaga, jortega@uma.es, felipe@uma.es.
}

\begin{abstract}
Among the many requirements imposed by the EHEA, one of the most concerning is to improve coordination among the subjects of a degree, and has become one of the liveliest topics of debate in the education community. On one hand, we can find lecturers which are teaching subjects for students with noticeable gaps in some content, while the students, in turn, not only have to fill these gaps with extra effort, but they also need to deal with numerous content duplications, stealing their time and effort, and undermining the quality of their training. One cause of these defects is the speed in the implementation of degrees, possibly coupled with a lack of resources on the part of those responsible for drawing up the plans.

This paper presents a set of Web applications aimed at drawing up a map of dependencies between degree subjects at University. Based on a survey system with micro-polls, a database of relationships among subjects and a web system has been design. These tools can detect and report existing failures in academic coordination, thus providing a valuable tool to improve the consistency and quality of the degree curricula.
\end{abstract}

Keywords: Vertical coordination, academic survey, dependency graph, web applications.

\footnotetext{
Resumen

Entre las múltiples exigencias que impone el EEES, la mejora de la coordinación entre las asignaturas de una titulación es una de las que más preocupan, y se ha convertido en uno de los temas de debate más vivos en la comunidad educativa. Por una parte los docentes nos hemos encontrado impartiendo asignaturas a un alumnado con notorias carencias en algunos contenidos, mientras que ellos, por su parte, no sólo tienen que suplir dichas
} 
carencias con esfuerzo adicional, sino que además se encuentran con numerosas duplicidades de contenidos que restan tiempo y calidad a su formación. Una de las causas de estos defectos es la celeridad en la implantación de titulaciones, unido posiblemente a una falta de recursos por parte de los responsables de la elaboración de los planes.

Este trabajo presenta un conjunto de aplicaciones web orientadas a la elaboración de un mapa de dependencias entre las asignaturas de una titulación. Basándose en un sistema de encuestas, se ha implementado una base de datos de dependencias y un sistema web que permiten detectar $e$ informar de los defectos de coordinación existentes, proporcionando una herramienta de gran valor para mejorar la coherencia y la calidad de los planes de estudios.

Palabras clave: Coordinación vertical, encuesta académica, grafos de dependencias, aplicaciones web

\section{Introducción}

Una de las reclamaciones más frecuentes en el alumnado de las titulaciones universitarias es que exista una coordinación adecuada entre las asignaturas de una misma titulación para que el currículum académico proporcione al alumno una adecuada formación sin la necesidad de que éste tenga que realizar un esfuerzo adicional debido a una inadecuada elaboración e implantación del plan docente. Entre los problemas de coordinación más frecuentes, y muy por encima de otras causas, se encuentran la repetición de contenidos y la falta de los conocimientos básicos que requieren las asignaturas. En la mayoría de los casos en España, estos problemas están derivados de una implantación excesivamente rápida de nuevas titulaciones para adaptar nuestro sistema al EEES, así como las posteriores modificaciones que estamos sufriendo. La celeridad es una mala compañera de viaje en el diseño de un currículum, pero no es la única causa de los defectos de coordinación. Algunas de ellas son inevitables: No podemos exigir un conocimiento absoluto de los contenidos al equipo responsable de la organización académica, y más aun considerando que las materias impartidas evolucionan tan rápido como lo hace la sociedad (Valero, 2007). Tampoco resulta técnicamente posible que todos los implicados puedan haber participado en la elaboración y depuración de los planes de estudio, como sería deseable (Costa, 2011).

Resulta pues necesario pulir constantemente los planes de estudio - especialmente en los primeros cursos de una titulación- ya sea para corregir sus defectos o por pura evolución, y en consecuencia, hacen falta herramientas que permitan que el mayor número de actores implicados estén presentes en este proceso de mejora. En este trabajo presentamos una 
herramienta que ha demostrado ser extremadamente útil para cumplir estos objetivos generales: un sistema de microencuestas a la comunidad universitaria que permite identificar los principales problemas de coordinación vertical entre las asignaturas de titulaciones de Grado.

\section{Objetivos concretos}

El objetivo de este trabajo es el desarrollo de un conjunto de aplicaciones centradas en la web y que están orientadas a la elaboración de un mapa de dependencias entre las asignaturas de una titulación. Basándose en un sistema de encuestas vía Web, se pretende implementar una base de datos de dependencias, junto a un sistema de información automatizado, que permitan $1^{\circ}$ ) detectar los defectos de coordinación existentes, $2^{\circ}$ ) notificar a los docentes implicados, y $3^{\circ}$ ) generar información sobre las dependencias mediante un gráfico con comentarios. Esta representación gráfica de las relaciones entre asignaturas, puesta en manos de los organismos y equipos responsables de la ordenación y coordinación académica, debe suponer una herramienta de gran valor para alcanzar objetivos que garanticen la coherencia y la calidad de los planes de estudios.

Los objetivos concretos del sistema de coordinación propuesto son:

- Diseñar un sistema de recogida de información a través de la web (encuestas).

- Revisar y contrastar que la secuenciación de asignaturas y contenidos en el conjunto de materias afines es adecuada, mediante un análisis de los resultados de las encuestas.

- Analizar los contenidos de las asignaturas del plan de estudios para evitar lagunas y solapes, y con ello, optimizar la carga de trabajo del alumno.

- Proporcionar al alumnado un diagrama de dependencias de asignaturas que le ayude en la preparación de sus estudios y configurar adecuadamente su propio curriculum.

\section{Desarrollo de la innovación}

El elemento más importante de la herramienta diseñada es el formulario con la encuesta para la captura de datos. La simplicidad del formulario garantiza una alta participación entre el alumnado, y por esta razón, en el formulario sólo es necesario elegir las asignaturas afectadas, un selector que indica el tipo de problema (contenidos que no se explican, contenidos que se repiten o simple dependencia) y un campo de texto libre en el que se puede describir el problema con el nivel de detalle deseado. Dicho formulario es diferente, en función del perfil del encuestado (Por ejemplo, el profesor responsable de una asignatura

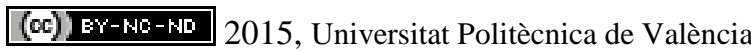


no debería “denuncia” que está repitiendo contenidos en su propia asignatura. En la Figura 1 se muestra una captura de pantalla de la hoja de encuestas.

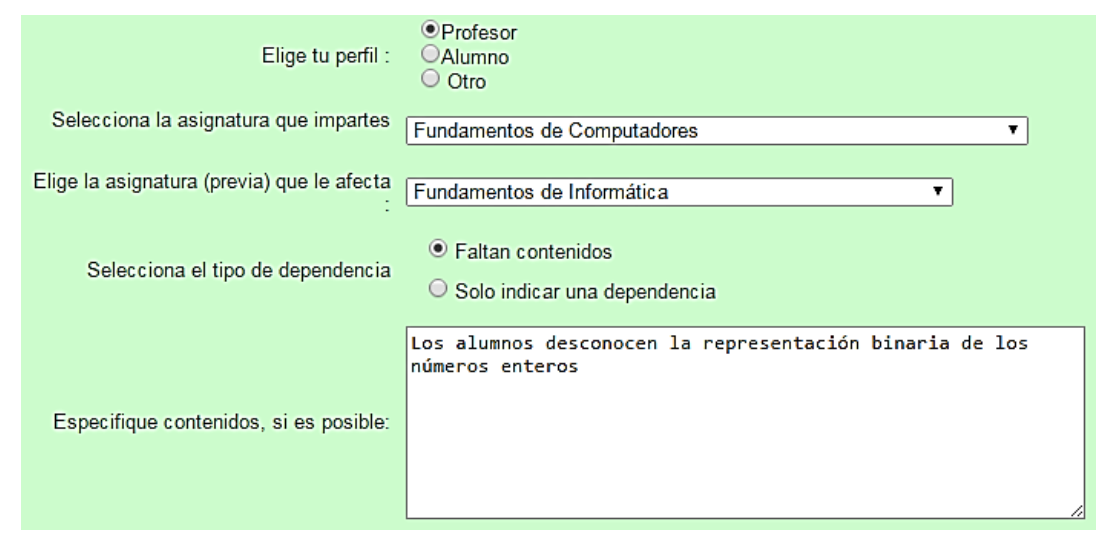

Fig. 1 Captura de pantalla de la hoja de encuestas

Los resultados de las encuestas, en el mismo momento del envío, son almacenados en una base de datos mysql.

\subsection{Generación del Sistema de Información}

Aunque cada una de las encuestas almacenadas en la base de datos apenas proporciona información útil para la coordinación, es la suma de microencuestas la que en conjunto, genera una herramienta de excepcional utilidad. Esta idea ha sido utilizada con cierta frecuencia en otros campos de ciencia e ingeniería (Agarwall, 2004; Steinle, 2006, entre otros), incluso para la elaboración de grafos de dependencias entre sistemas informáticos (Chandra, 2008), pero no tenemos referencia de que nunca se haya utilizado para la depuración de la ordenación docente. Evidentemente, un inadecuado procesamiento de los datos podría generar un producto inútil, por lo que esta parte del proyecto resulta crítica. Con esa idea subyacente, se ha programado un sitio Web de consulta de los resultados de las encuestas en el que se pongan de manifiesto los principales problemas de coordinación detectados. Igualmente se ha diseñado un sistema de notificaciones para que los coordinadores de asignatura reciban información puntual de los resultados. Estas notificaciones no se envían de forma automática, sino que requieren de la figura del coordinador (o equipo de coordinación) académico de la titulación, como parte de un proceso de filtrado. 


\subsection{Visualización gráfica de resultados}

También resulta imprescindible que la información generada sea atractiva y fácilmente accesible para que los docentes y alumnos implicados participen activamente. Para ello se han generado diagramas de dependencias entre las asignaturas con todo tipo de detalles (orientados a un curso, sobre una asignatura, en un semestre o un área de conocimiento, entre otros). Para la generación de las gráficas de dependencias se ha programado un servicio encargado de consultar la base de datos y generar una descripción de las dependencias de las asignaturas en el lenguaje intermedio de descripción de grafos dot. Un segundo servicio genera las imágenes jpg que serán incorporados al sistema de información. La Figura 5 muestra un ejemplo gráfico. Este grafo incorpora las dependencias de las asignaturas del área de Mecánica de Fluidos.

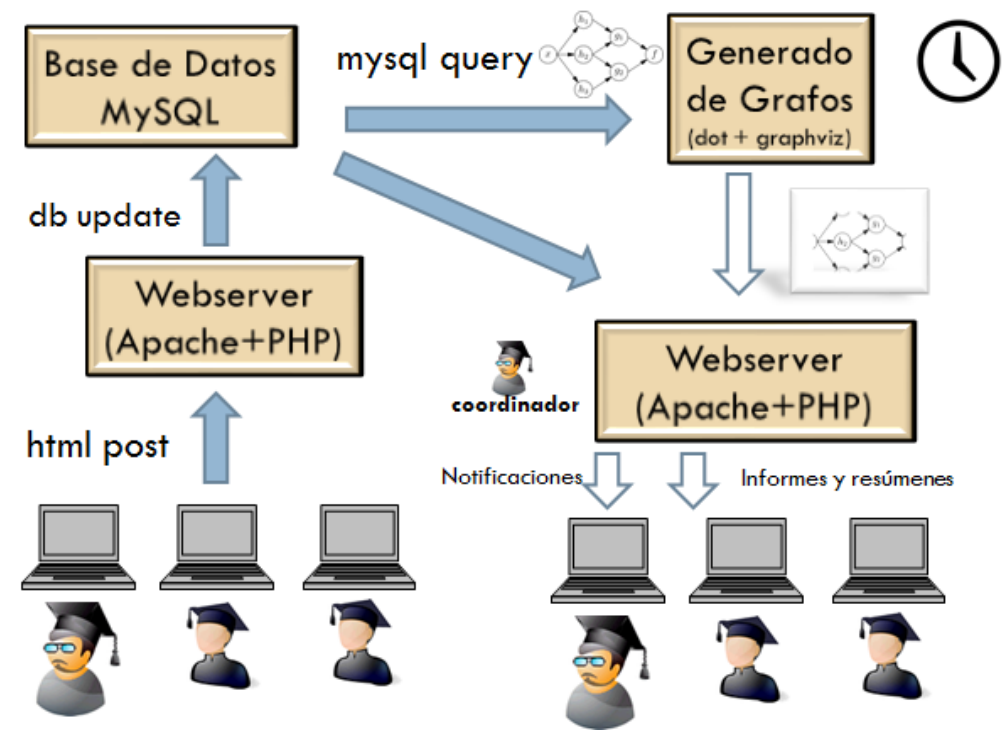

Fig. 2 Estructura del Sistema de Información

\subsection{Instalación e implantación del sistema}

Una vez diseñado el sistema (cuya estructura se representa en la Figura 2) es necesario planificar adecuadamente su puesta en marcha no solo en una, sino en varias titulaciones. En este proyecto se ha programado una implantación progresiva para evitar no sólo un colapso de las plataformas web sino también para facilitar el perfeccionamiento de la

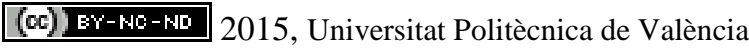


herramienta. En una primera fase, se ha comunicado la existencia de los formularios a un número restringido de participantes (representantes de alumnos y coordinadores de las titulaciones). La captura de un conjunto inicial de datos ha permitido detectar las posibles deficiencias y la consecuente revisión de la misma. En fases posteriores se ha ampliado el número de personas informadas de la existencia de la web, hasta que todos los alumnos de una misma titulación (el Grado de Ingeniería de Tecnologías Industriales, GITI, de la Universidad de Málaga) han sido informados.

La última fase implementada ha consistido en extender la herramienta a otras titulaciones de diferentes universidades andaluzas. Para ello se ha diseñado, dentro del sitio web, un formulario de suscripción que destaca por su simplicidad (Figura 3). El propio sitio, mediante el lenguaje de programación PHP se encarga de preparar todo el sistema de forma que el suscriptor sólo necesita informar a su comunidad de la URL de la máquina virtual que la implementará.

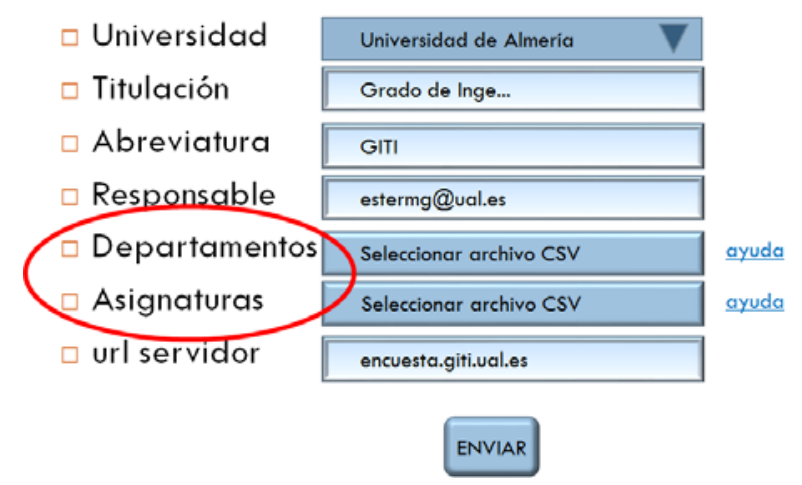

Fig. 3 Herramienta para la suscripción al sistema de nuevas asignaturas

Actualmente el sistema está disponible y se le ha dado suficiente divulgación en varias titulaciones de distintas universidades andaluzas.

\section{Resultados}

Desde el mismo momento de la implantación del sistema, alumnos y docentes tienen acceso a los datos de las encuestas. En el caso de los docentes, el acceso es completo, por lo que pueden consultar la descripción exhaustiva de las microencuestas, mientras que los alumnos sólo tienen acceso a la representación gráfica de los resultados. En las Figuras 4 y 5 se muestran algunos detalles del sistema de información. En particular, el diagrama de 
dependencias de la Figura 5 muestra el resultado de casi un centenar de microencuestas en las que se referencia alguna asignatura (del mencionado grado de GITI) del área de conocimiento de Mecánica de Fluidos de la Universidad de Málaga.

Como detalle, señalar que los arcos del grafo indican la dependencia entre dos asignaturas, siendo su grosor proporcional al número de encuestas que determinan dicha dependencia. Además, un código de colores determina también qué tipo de problemas se producen. Así, en color rojo se indica la carencia de contenidos, mientras que el verde se utiliza para indicar contenidos que se repiten.

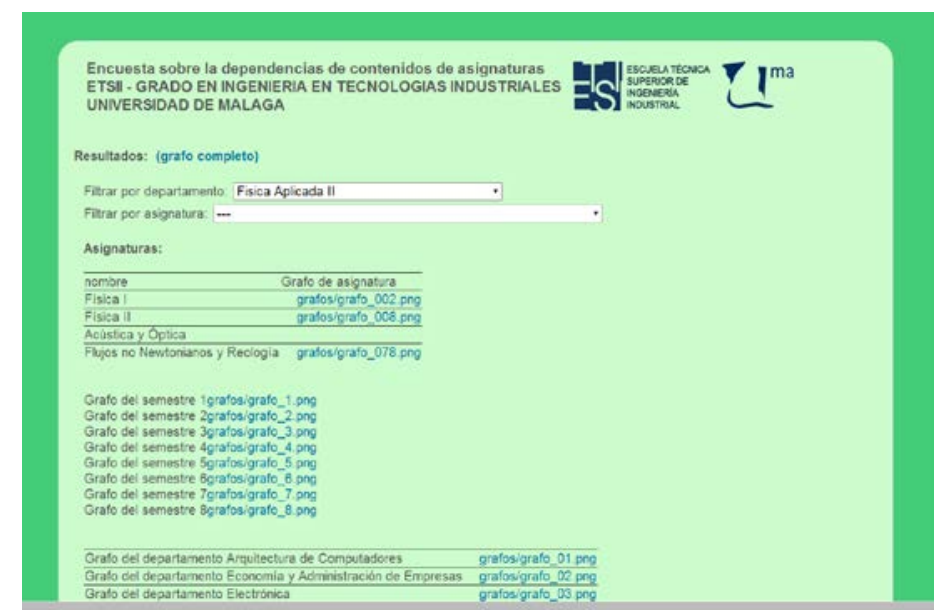

Fig. 5 Detalle del sistema de información de resultados

Además del producto generado (la propia herramienta web), el verdadero objetivo del proyecto es comprobar la verdadera utilidad. En este sentido, los resultados no sólo demuestran que los objetivos del proyecto se han alcanzado sino que han ido más allá de lo inicialmente esperado:

En distintas reuniones de los equipos de coordinación con los alumnos de diferentes cursos — siguiendo el modelo propuesto de implantación por fases - se les ha ido informando sobre este proyecto. Los alumnos no solo han acogido muy favorablemente esta iniciativa sino que además han sugerido las modificaciones necesarias para que tanto alumnos como profesores puedan sacar el máximo provecho a los resultados de las encuestas. Entre estas modificaciones, la más significativa es que el alumno puede manifestar (en el mismo portal de encuestas) las deficiencias que pueden encontrar en los contenidos o en la docencia de una asignatura, sin necesidad de que haya dependencias con otras asignaturas.

\section{(cc) EY-NC-ND 2015, Universitat Politècnica de València}




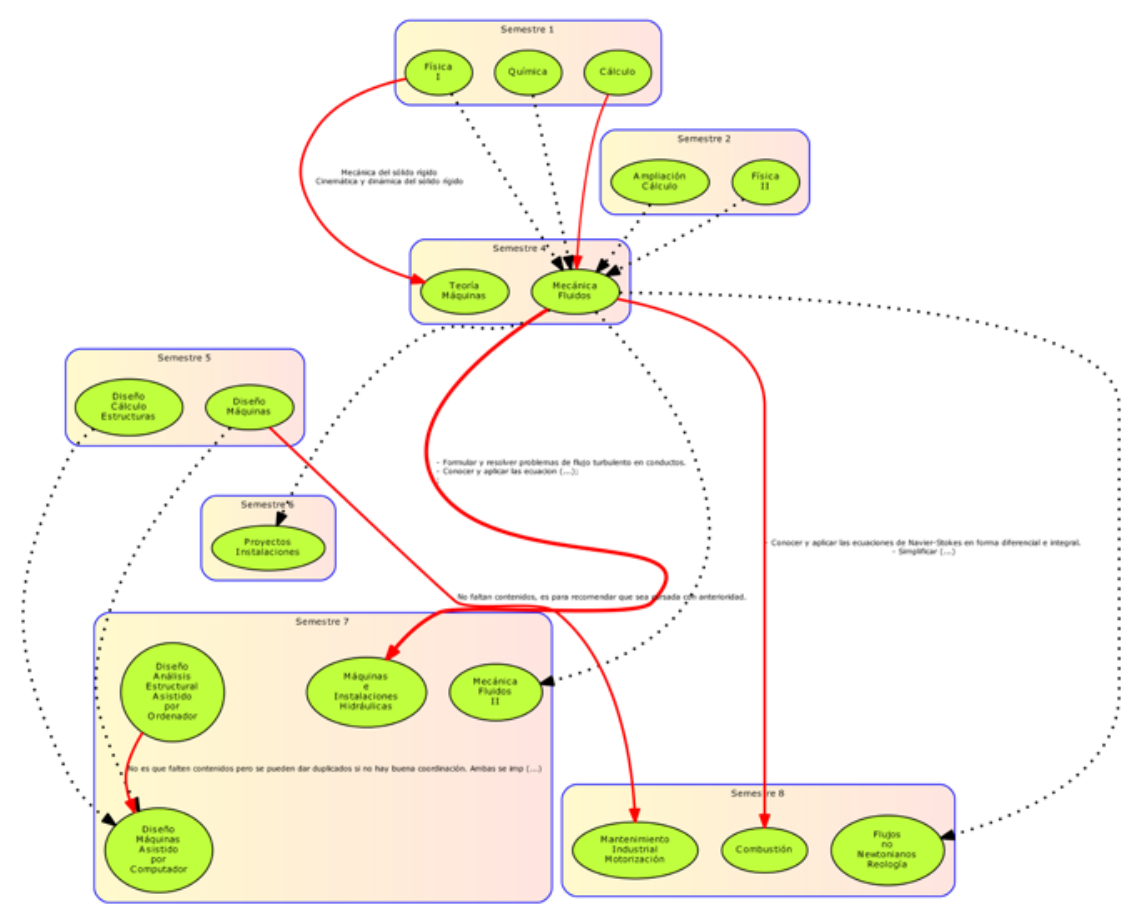

Fig. 5 Grafo de dependencias relativo a las asignaturas de un departamento en una titulación

Algo parecido ha ocurrido en las reuniones con los profesores, aunque es cierto que hay más reticencias puntuales. A pesar de ello, la acogida ha sido en general muy favorable.

Los resultados de las encuestas han detectado deficiencias de coordinación que se han notificado a los profesores implicados, dejando completa autonomía a los mismos para la resolución de las mismas.

\section{Conclusiones}

En este trabajo se ha desarrollado un sistema de encuestas, realizadas vía web, mediante el cual los miembros de la comunidad universitaria pueden informar sobre posibles dependencias entre asignaturas, así como defectos puntuales de coordinación académica que hayan detectado en una misma titulación.

El conjunto de encuestas obtenidas en cada titulación se ha utilizado para la elaboración de un mapa de dependencias en el que se ponen de manifiesto no sólo las relaciones entre asignaturas, sino también las duplicidades de contenidos, las carencias o cualquier otro tipo

(c)) EY-NC-ND 2015, Universitat Politècnica de València 
de problemas de carácter académico. Dicho mapa ha demostrado ser una herramienta de gran valor para la mejora de los programas académicos y el seguimiento de su cumplimiento.

\section{Agradecimientos}

Los autores quieren mostrar su reconocimiento a la Universidad de Málaga por la financiación de este trabajo mediante el Proyecto de Innovación Educativa PIE13-014 denominado "Sistema Web para la mejora de la Coordinación Vertical en Titulaciones Universitarias”.

\section{Referencias}

AGARWAL, M.K. ; GUPTA M. ; KAR, G., NEOGI, A. y SAILER, A. (2004) "Mining Activity Data for Dynamic Dependency" en Discovery in e-Business Systems., IEEE Transactions on Network and Service Management, Vol. 1 , issue 2, p. 49-58

COSTA P. ; PERIAGO C. y GARCÍA BAÑO, P. (2011) "Estrategias de coordinación horizontal y vertical en los planes de estudios adaptados al EEES”, Congreso Internacional de Innovación Docente, Universidad Politécnica de Cartagena.

CHANDRA, B.P. ; MALTZ, D.A. ; NATH, S. Y ZHANG, M. (2008) "Managing Networks Using Dependency Analysis”, US Patent, Referencia US 20080016115 A1.

STEINLE, M. ; ABERER, K.; GIRDZIJAUSKAS, S. y LOVIS, C (2006) "Mapping Moving Landscapes by Mining Mountains of Logs: Novel Techniques for Dependency Model Generation" Proceedings of the 32nd international conference on Very large databases VLDB '06, p. 1093-1102

STARK, J.S. (2000). "Planning introductory college courses: Content, context and form" en Instructional Science, vol. 28, issue 5, p. 413-438.

STARK, J.S. y LATTUCA L.R. (2011) Shaping the College Curriculum: Academic Plans in Context, New York, John Wiley \& Sons.

TSINIDOU, M.; GEROGIANNIS, V. Y FITSILIS, P. (2010) "Evaluation of the factors that determine quality in higher education: an empirical study" en Quality Assurance in Education , vol. 18, issue 3, p. 227-244

VALERO M. (2007) ¿Por qué el EEES nos obliga a coordinarnos más y mejor? Universidad Politécnica de Cataluña.

$<$ http://epsc.upc.edu/projectes/usuaris/miguel.valero/materiales/docencia/conferencias/Almeria.pdf> [Consulta: 28 de mayo de 2015]

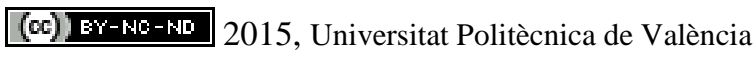

Congreso In-Red (2015) 